\title{
Occludin is required for apoptosis when claudin-claudin interactions are disrupted
}

\author{
N Beeman ${ }^{1}$, PG Webb ${ }^{1}$ and HK Baumgartner,
}

Disruption of tight junctions is often seen during pathogen infection, inflammation, and tumor progression. Mislocalization of the tight junction proteins occludin and claudin in mammary epithelial monolayers leads to apoptosis through the extrinsic pathway. To further investigate the mechanism of this response, a normal mammary epithelial cell line $(\mathrm{EpH} 4)$ as well as primary mammary epithelial cells were treated with a claudin-disrupting mimic peptide, DFYNP (aspartic acid-phenylalanine-tyrosine-asparagineproline). Using fluorescent indicators, we found that caspase-3 activation, resulting from treatment with DFYNP, was restricted to $\mathrm{EpH} 4$ and primary mammary epithelial cells with mislocalized claudin-4. Mislocalized claudin-4 and occludin were colocalized in non-junctional puncta, and both molecules were found in the death-inducing signaling complex (DISC) where they colocalized with Fas, fas-associated protein with death domain (FADD), active caspase-8 and caspase-3 at distinct apical domains. Importantly, caspase-3 activation was totally repressed in primary mammary epithelial cells from occludin null mice. Thus, the apoptotic response appears to be initiated by the movement of occludin to the DISC suggesting that this molecule has signaling properties that initiate cell death when its tight junction location is disrupted.

Cell Death and Disease (2012) 3, e273; doi:10.1038/cddis.2012.14; published online 23 February 2012

Subject Category: Cancer

Tight junction disruption has an important role in several pathologies including viral and bacterial infection, ${ }^{1,2}$ inflammation, ${ }^{3,4}$ and tumor progression. ${ }^{5}$ Claudin and occludin are major transmembrane proteins of the tight junction that provide tight control over paracellular diffusion within an epithelium. Interestingly, claudin and occludin are often targeted and misplaced by viruses, ${ }^{1,6}$ bacteria, ${ }^{7,8}$ and inflammatory cytokines. ${ }^{8,9}$ Although apoptosis is observed in many diseases that involve disrupted tight junctions, ${ }^{10-12}$ the role displaced tight junction proteins may have in the initiation of apoptosis has not been investigated. Importantly, it is not known whether changes in localization of key tight junction proteins, such as claudin and occludin, may be initiating important signaling pathways to preserve the barrier properties of a compromised epithelial monolayer.

Previously, we have shown, using a mimic peptide to a conserved sequence in the second extracellular loop of occludin, that disruption of normal occludin-occludin interactions at the tight junction leads to mislocalization of occludin away from tight junctions and into the cytosol. ${ }^{13}$ This mislocalized occludin interacted with components of the death-inducing signaling complex (DISC), such as fasassociated protein with death domain (FADD) and cleaved caspase-8, and induced activation of the extrinsic apoptotic pathway. This finding suggested that occludin not only has a role in barrier formation, but may also be a signaling molecule that induces apoptosis when normal tight junction protein interactions are disrupted. When normal claudin-claudin interactions were disrupted, using similar peptide mimic technology, claudin also mislocalized away from tight junctions into the cytosol. ${ }^{14}$ The mislocalization of claudin led to the activation of caspase- 8 and -3 , similar to what was seen when occludin was disrupted, and suggested that claudin may also be having an important role in cell fate signaling in response to tight junction disruption.

In the present study, the signaling mechanism involved in apoptosis induced by claudin and occludin disruption was further investigated. We asked whether both proteins work through the same pathway or whether one protein is a universal trigger for apoptosis when tight junctions are disrupted. Normal mammary epithelial cells were treated with a claudin mimic peptide, DFYNP (aspartic acid-phenylalanine-tyrosine-asparagine-proline), and changes in localization of claudin and occludin were examined in relation to each other as well as to key apoptotic signaling molecules. The results show that both claudin and occludin move from tight junctions to distinct apical domains where they colocalize with components of the DISC to induce apoptosis. However, it is occludin that is required for induction of apoptosis.

\section{Results}

Apoptosis is restricted to epithelial cells with mislocalized claudin. To confirm that disruption of normal

\footnotetext{
${ }^{1}$ Department of Physiology and Biophysics, University of Colorado Denver, School of Medicine, 12700 East 19th Avenue, Aurora, CO 80045, USA

${ }^{*}$ Corresponding author: HK Baumgartner, Department of Physiology and Biophysics, University of Colorado Denver, School of Medicine, Reproductive Sciences/ Mail Stop 8613, 12700 East 19th Avenue, Room 3400, Aurora, CO 80045, USA. Tel: + 1303724 3502; Fax: + 1303724 3512; E-mail: Heidi.Wilson@ ucdenver.edu Keywords: claudin; occludin; apoptosis; death-inducing signaling complex (DISC)

Abbreviations: DFYNP, aspartic acid-phenylalanine-tyrosine-asparagine-proline; DISC, death-inducing signaling complex; DMEM/F12, Dulbecco's modified Eagle medium: nutrient mixture F-12; FADD, fas-associated protein with death domain; FBS, fetal bovine serum; $\mathrm{H}$. pylori, Helicobacter pylori; LYHY, lysine-tyrosinehistidine-tyrosine; PBS, phosphate buffered saline

Received 11.11.11; revised 31.1.12; accepted 31.1.12; Edited by A Stephanou
} 
cleaved casp-3
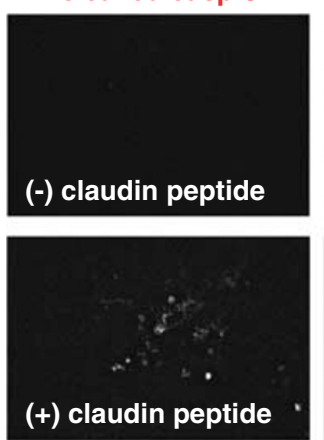

claudin-4
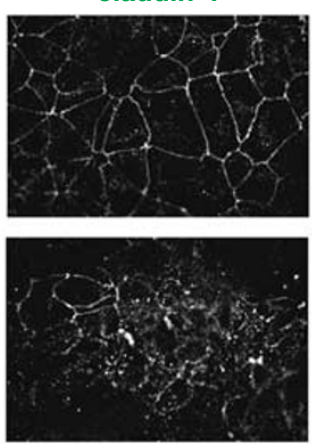

DAPI
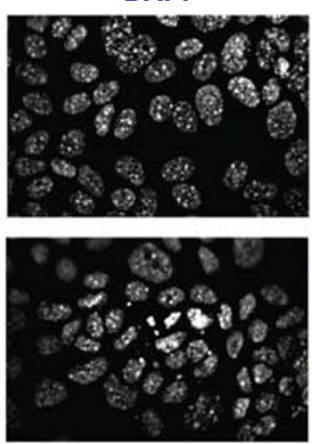

overlay
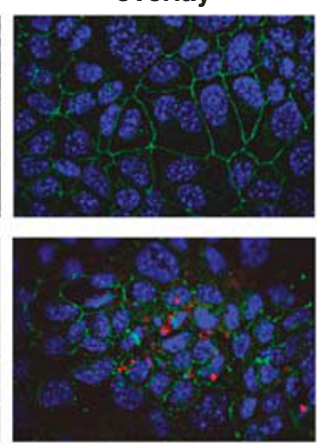

Figure 1 Caspase-3 is activated in cells with mislocalized claudin. Confocal microscopy images show staining of EpH4 mammary epithelial monolayers for active caspase-3 (red), claudin-4 (green), and nuclei (blue). Caspase-3 is activated in cells treated with the DFYNP claudin mimic peptide where activation is restricted to cells with non-junctional claudin-4 localization

claudin-4
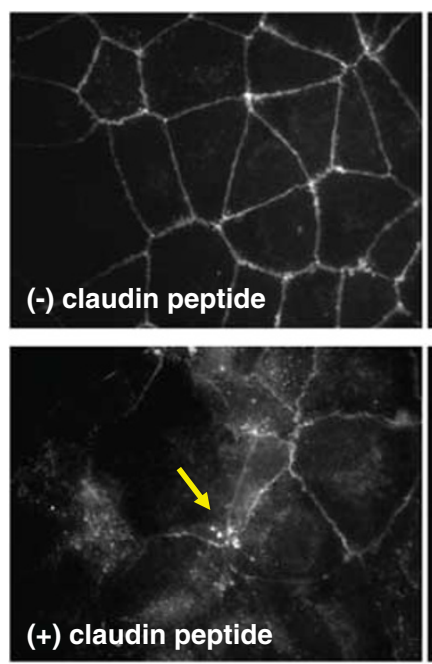

occludin
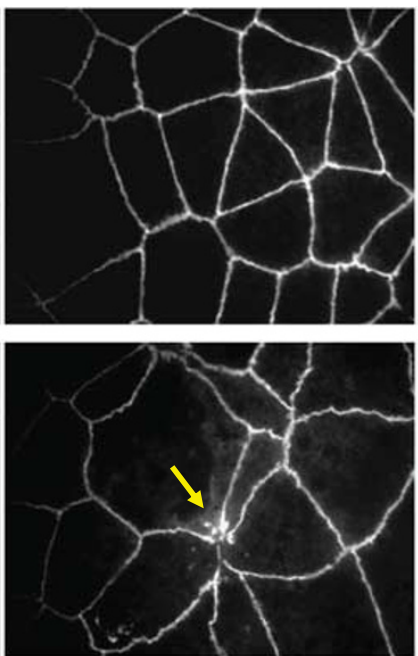

overlay
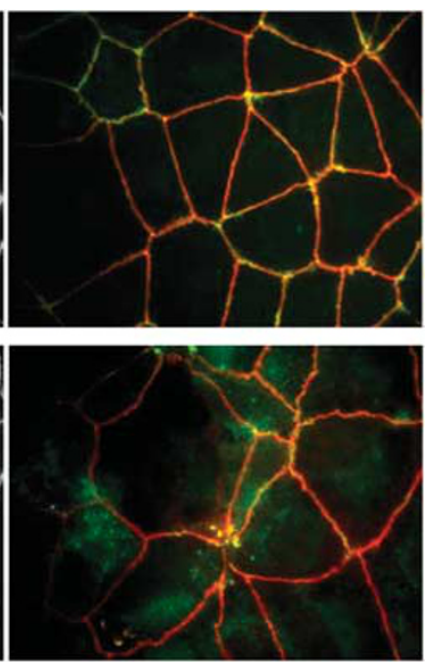

Figure 2 Colocalization of occludin and claudin. Confocal microscopy images show staining of EpH4 mammary epithelial monolayers for claudin-4 (green) and occludin (red) in the absence and presence of the claudin mimic peptide. Claudin-4 and occludin both localize to sites of tight junctions in untreated epithelium. After treatment with the claudin mimic peptide, bright puncta of non-junctional occludin colocalize with bright puncta of non-junctional claudin-4

claudin localization leads to apoptosis, cells were examined for localization of both claudin-4 and active caspase- 3 in the absence and presence of the claudin-disrupting DFYNP mimic peptide. Immunohistochemistry, using antibodies directed to claudin-4 (a claudin subtype that contains DFYNP in the second extracellular loop) and cleaved caspase-3, revealed that claudin-4 is largely restricted to tight junctions and caspase- 3 was not activated in untreated $\mathrm{EpH} 4$ mammary epithelial cells (Figure 1). After $16 \mathrm{~h}$ of treatment with the claudin mimic peptide, claudin-4 localization changed dramatically, with a significant proportion of claudin in the cytosol. Treated cells also showed activated caspase-3.

Occludin is disrupted when claudin is mislocalized. As occludin mislocalization can also lead to apoptosis, ${ }^{13}$ we wanted to test whether or not occludin is disrupted in response to claudin mislocalization. Claudin-4 and occludin localization were examined simultaneously in the absence and presence of the claudin mimic peptide. In untreated EpH4 cells, both claudin-4 and occludin are restricted to tight junctions (Figure 2). After $16 \mathrm{~h}$ of treatment with the claudin mimic peptide, disruption of claudin-4 was very clear in defined groups of treated cells where claudin- 4 appeared to be less regularly distributed in the tight junctions than in controls. Occludin was also mislocalized into non-junctional puncta in treated $\mathrm{EpH} 4$ cells, but there was still a strong presence of occludin at the tight junction. Interestingly, bright puncta of non-junctional occludin colocalized with nonjunctional puncta of claudin-4. This observation suggests occludin is also mislocalized in response to claudin disruption and interacts with claudin in distinct vesicular-like domains.

Claudin and occludin colocalize with components of the DISC. The extrinsic apoptotic pathway is characterized by the activation of a death receptor and formation of the DISC, of which FADD and cleaved caspase- 8 are key components, and initiation of the caspase cascade. EpH4 cells treated with 
a
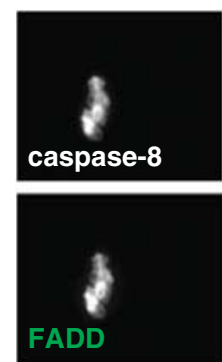

c
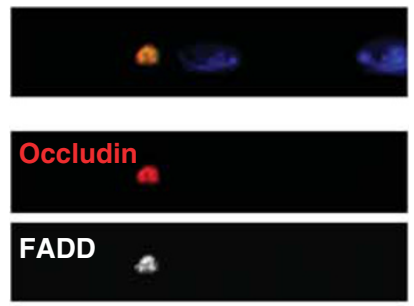

Caspase-8 b
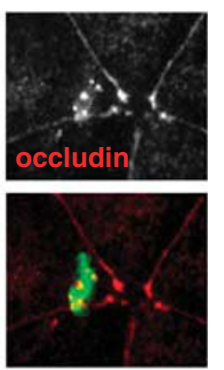

d
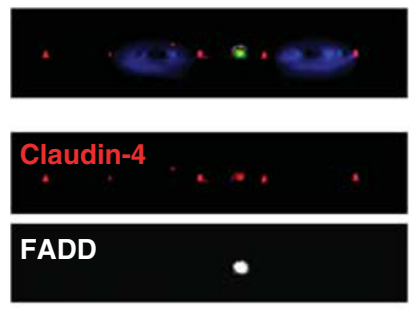

Caspase-8

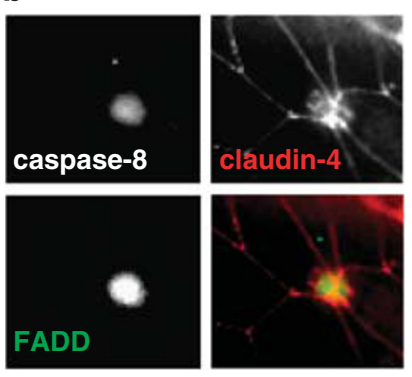

e
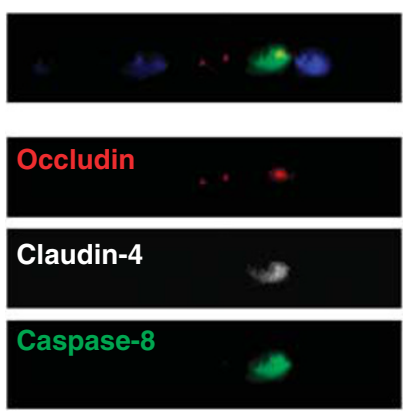

Figure 3 Colocalization of occludin, claudin-4, and components of the death inducing signaling complex (DISC). Magnified $(\times 100)$ confocal microscopy images show staining of EpH4 mammary epithelial monolayers for: (a) occludin (red), active caspase-8 (white), and FADD (green); (b) claudin-4 (red), active caspase-8 (white), and FADD (green) after treatment of cells with the claudin mimic peptide for $4 \mathrm{~h}$. Claudin-4 and occludin both colocalize with active caspase-8 and FADD in response to treatment with the peptide. Z-stack images show: (c) EpH4 cells stained for nuclei (DAPI, blue), occludin (red), FADD (white), and active caspase-8 (green); (d) cells stained for nuclei (blue), claudin-4 (red), FADD (white), and active caspase-8 (green); or (e) cells stained for nuclei (blue), occludin (red), claudin-4 (white), and active caspase-8 (green). Occludin, claudin-4, FADD, and active caspase-8 colocalize to distinct apical domains in response to treatment with the claudin mimic peptide

a
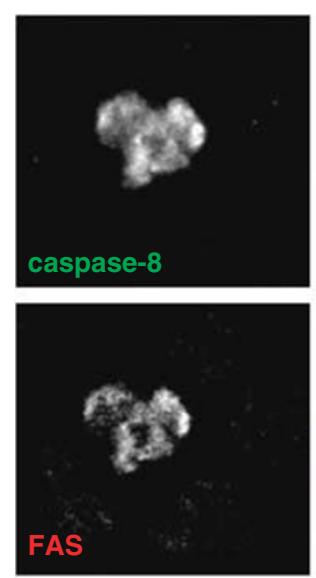
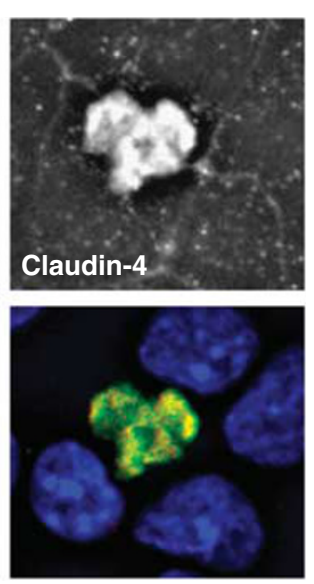

b
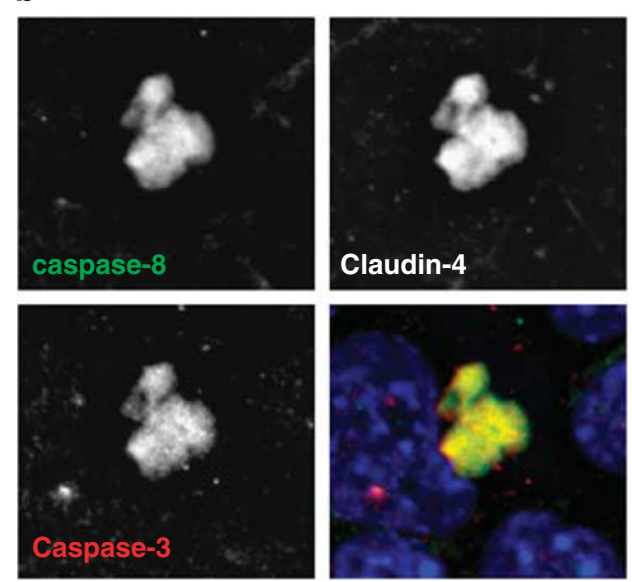

Figure 4 Fas and caspase-3 colocalize with claudin and DISC components. Magnified $(\times 100)$ confocal microscopy images show staining of EpH4 mammary epithelial monolayers for active caspase-8 (green), claudin-4 (white) and (a) Fas or (b) active caspase-3 (red) after $4 \mathrm{~h}$ of treatment with the claudin mimic peptide. Claudin- 4 colocalizes with active caspase-8 as well as Fas and activated caspase- 3 in response to peptide treatment

the claudin mimic peptide for $4 \mathrm{~h}$ showed non-junctional occludin colocalized with aggregated FADD and cleaved caspase-8 (Figures $3 a$ and $\mathrm{c}$ ). Immunohistochemistry also showed colocalization of claudin-4 with aggregated FADD and active caspase-8 (Figures $3 b$ and $d$ ). These data suggest that both occludin and claudin interact with the DISC in response to claudin disruption with the DFYNP mimic peptide. Figure $3 e$ confirms localization of both occludin and claudin at sites of DISC formation, with occludin and claudin-4 colocalizing with active caspase-8.
Interestingly, z-stack images of claudin and occludin with the DISC reveal restriction of claudin, occludin, FADD, and caspase-8 to an apical, vesicle-like domain within the cytoplasm near a tricellular junction (Figures $3 c-e$ ). The aggregation and activation of death receptors is thought to be upstream of FADD and caspase-8 recruitment into the DISC. Immunohistochemistry reveals that the Fas receptor is aggregated and also colocalized within the DISC formed in response to claudin disruption with the mimic peptide (Figure 4a). Downstream of procaspase-8 recruitment to 
a

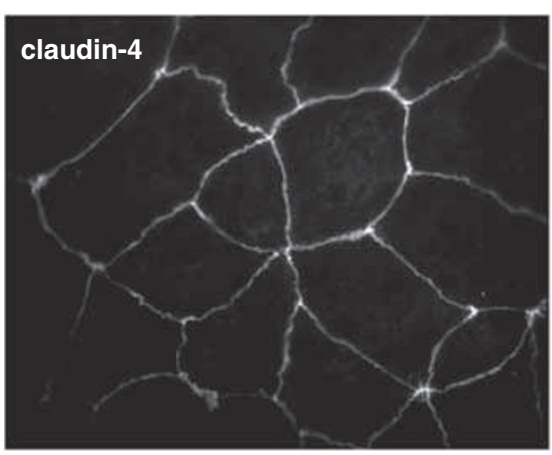

(-) occludin peptide b

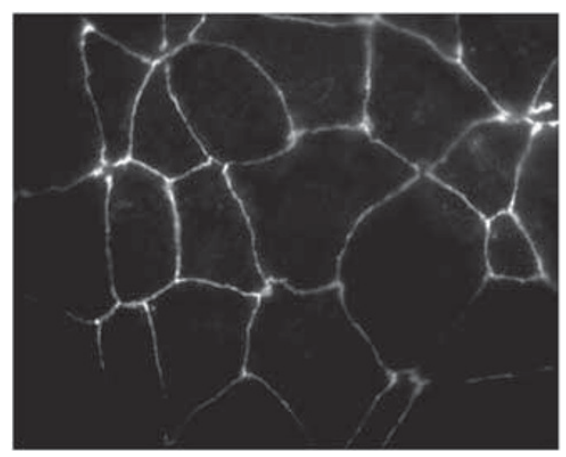

(+) occludin peptide

Figure 5 Claudin is not disrupted when occludin is disrupted. Confocal microscopy images show staining of EpH4 mammary epithelial monolayers for claudin-4 in the (a) absence and (b) presence of an occludin-disrupting LYHY mimic peptide. Claudin-4 remains at sites of tight junctions when occludin is disrupted

the DISC, and subsequent activation of caspase-8, is the activation of effector caspases, such as caspase-3, that carry out the enzymatic cleavage of other cellular proteins. Active caspase- 3 was also found within the DISC in response to claudin disruption with the mimic peptide (Figure 4b). Therefore, in response to claudin disruption, claudin and occludin move out of the tight junction and interact with the key protein components of the intrinsic apoptotic signaling pathway.

\section{Occludin disruption is downstream of claudin} mislocalization. The presence of occludin at the DISC when claudin localization is disrupted and caspase activation is induced further suggests that occludin may be having a key role in activation of apoptosis in response to tight junction disruption. To determine whether claudin and occludin act together to induce apoptosis when tight junctions are disrupted or whether occludin is the downstream sensor of tight junction disruption and trigger of apoptosis, we examined the localization of claudin during occludin disruption. Using immunohistochemistry, localization of claudin-4 was imaged after treatment with an occludindisrupting mimic peptide LYHY (lysine-tyrosine-histidinetyrosine). ${ }^{13}$ Figure 5 shows that claudin- 4 remained at the sites of tight junctions after treatment of the cells with the occludin-disrupting peptide. These data suggest that occludin, in fact, is acting downstream of claudin disruption to activate apoptosis and that occludin is more of a universal 'trigger' of apoptosis when tight junctions are disrupted.

Occludin is required for apoptosis induced by claudin disruption. To further test this hypothesis that occludin is the trigger of apoptosis when tight junctions are disrupted, we examined the ability of the claudin mimic peptide to induce apoptosis in the absence of occludin. Primary mammary epithelial cells were isolated from pregnancy day- 15 occludin null-transgenic mice and compared with primary mammary epithelial cells isolated from wild-type mice of the same background (FVB). Western blot analysis (Figure 6a) confirmed that the occludin protein was absent in tissue from the occludin null mouse. Next, we examined claudin-4 localization in the occludin knockout mammary epithelial cells to determine whether claudin-4 localizes to the tight junctions in the absence of occludin. Results show that claudin-4 is, indeed, localized to the tight junctions in the occludin knockout mammary epithelium (Figure 6b). After treatment with the claudin mimic peptide, claudin-4 localization was significantly disrupted (Figure 6c), similar to what we saw with the EpH4 cell line. Next, the ability of the peptide to induce apoptosis in the absence of occludin was examined. Wild-type mammary epithelial cells treated with the claudin mimic peptide showed a significant increase in activated caspase-3 $(8.09 \pm 0.48 \%$ of cells, $P=0.0000169$ versus control) versus untreated wild-type control cells $(1.39 \pm 0.21 \%$ of cells, Figure 7$)$. Claudin disruption did not induce apoptosis in occludin null mammary epithelial cells, showing a similar level of caspase-3 activation $(1.59 \pm 0.16 \%$ of cells, $P=0.82013$ versus control) as untreated wild-type control cells. Interestingly, although claudin-4 was mislocalized, the change in its localization was unable to elicit the apoptotic response. This observation provides strong evidence that occludin mediates the apoptotic response induced by disruption of tight junction proteins.

\section{Discussion}

Tight junction disruption is usually thought of as a downstream consequence of caspase cleavage during the apoptotic process. Results from the present study, however, suggest that tight junction disruption can be an early event that initiates caspase activation and cell death. Previously, we have shown that disruption of the normal tight junctional localization of occludin $^{13}$ and claudin ${ }^{14}$ with mimic peptides can induce apoptosis in normal epithelium. Immunoprecipitation along with immunohistochemistry confirmed that occludin physically interacts with the DISC via the adaptor molecule FADD. ${ }^{13}$ In the present study, we have demonstrated that both occludin and claudin can interact with components of the DISC, but that occludin is required for the induction of apoptosis. Therefore, it appears that occludin is having an important role in sensing tight junction disruption and initiating apoptosis, a highly regulated form of cell death. As apoptotic cells can be extruded 


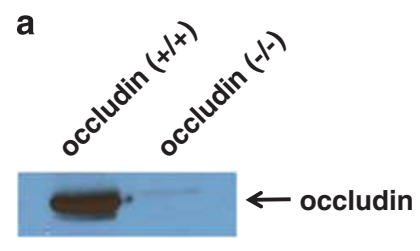

b

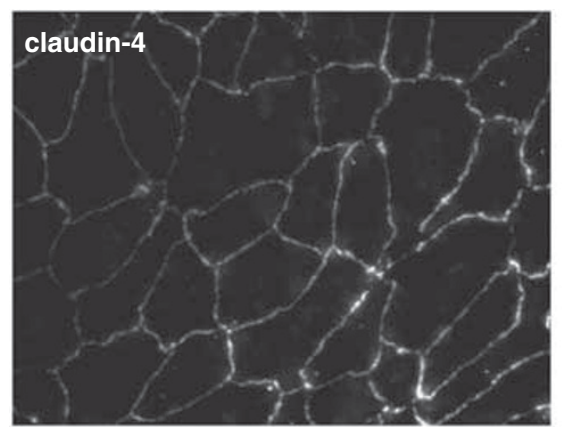

(-) claudin peptide c

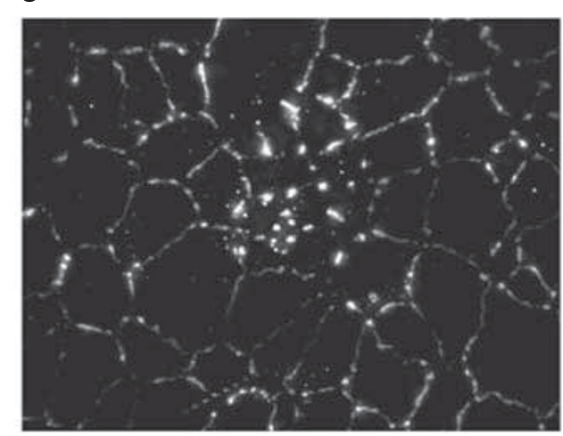

(+) claudin peptide

Figure 6 Claudin localization is disrupted in occludin null cells. (a) Western blot analysis of occludin protein expression was performed with tissue lysates from wild-type $(+I+)$ and occludin null $(-I-)$ mice to confirm the loss of occludin protein in occludin null cells. Confocal images show cultured monolayers of primary mammary epithelial cells isolated from occludin null mice stained for claudin-4 in the (b) absence and (c) presence of the claudin mimic peptide for $16 \mathrm{~h}$ before fixation. In the absence of occludin, claudin-4 is still able to change localization in response to the claudin mimic peptide

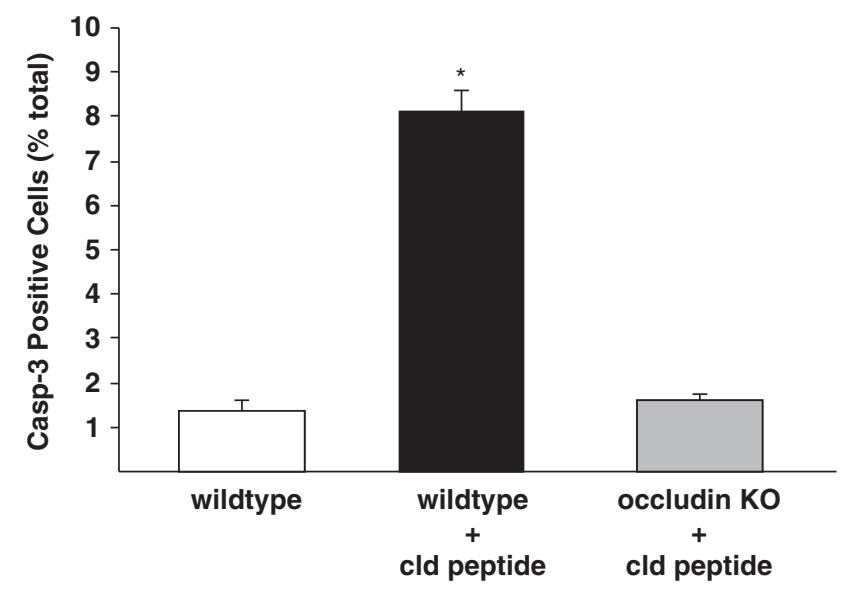

Figure 7 Occludin is required for peptide-induced caspase-3 activation. Cultured monolayers of primary mammary epithelial cells from wild-type and occludin null-transgenic mice were treated with the claudin mimic peptide. After $16 \mathrm{~h}$, the number of caspase-3-positive cells was measured. Caspase-3 activation in response to the claudin mimic peptide was inhibited in cells that lacked occludin protein expression. Mean \pm S.E.M., $n=3-6,{ }^{*} P<0.05$ versus untreated

from the epithelial monolayer with no change in epithelial resistance, a regulated cell death response to compromised cell junctions would provide a protective mechanism for maintaining barrier properties within an epithelium.

The implication that occludin may have a role outside of tight junction barrier formation can be inferred from the first experiments with occludin knockout mice: normally functioning tight junctions can form in the absence of occludin. ${ }^{15}$ The hypothesis that occludin has an important role in cell death signaling is supported by the results of the current study that show apoptosis is inhibited when occludin is absent. A hallmark characteristic of tumor cells is that they are resistant to apoptosis. That occludin can have a key role in apoptosis is supported by the reported loss of occludin expression in endometrial ${ }^{16}$ and breast ${ }^{17}$ carcinomas. Interestingly, Osanai et $a{ }^{18}{ }^{18}$ have shown that forced expression of occludin in a cervical cancer cell line (HeLa) that does not express occludin increased sensitivity of these cells to oxidative stress-induced apoptosis, further implicating occludin in apoptotic signaling.

The mechanism by which tight junction disruption leads to the interaction of occludin and claudin with the extrinsic apoptotic signaling pathway is not fully understood. Previously, we have shown that occludin can bind to FADD when tight junctions are disrupted. ${ }^{13}$ It is possible that displaced occludin is able to interact with FADD by movement within or between plasma membrane domains. Tight junction proteins, including occludin, have been found in cholesterol-rich lipid raft-like microdomains in the plasma membrane. ${ }^{19,20}$ The Fas death receptor has also been found in lipid raft domains, being recruited to lipid rafts upon stimulation, and further recruiting FADD and procaspase-8 for assembly of the death inducing signaling complex. ${ }^{21,22}$ Whether or not occludin is having a role in recruiting FADD downstream of Fas stimulation is unknown. However, Algeciras-Schimnich et al. ${ }^{21}$ have shown that FADD and procaspase-8 recruitment to the DISC and activation of caspase- 8 is required for clustering of FAS at the plasma membrane and internalization of the DISC. Therefore, it is possible the occludin interaction with FADD may be the initiating factor for DISC assembly, followed by clustering of Fas and internalization of the entire complex. It is also possible that the complex is responsible for the distinct vesicle-like apical domains seen in the z-stack images in Figure 3. 
Tight junction disruption, including occludin and claudin mislocalization, has been observed in Crohn's disease, ${ }^{12,23}$ hepatitis C virus infection, ${ }^{1,10}$ Helicobacter pylori infection, ${ }^{8,11,24}$ and many epithelial-derived tumors. ${ }^{5}$ Each of these disorders is associated with either upregulation of apoptosis, as seen in Crohn's disease, hepatitis $C$ virus, and $H$. pylori infection, ${ }^{12}$ or inhibition of apoptosis, as seen in breast cancer. ${ }^{17}$ It is a possibility that the signaling pathway identified in the current study may be contributing to the pathology of these diseases, either being upregulated to create a leaky epithelium or disrupted to create resistance to regulated cell death. Understanding the interaction of tight junction proteins with apoptotic signaling molecules will not only help to elucidate the pathology of these diseases but may provide potential therapeutic targets for their treatment.

\section{Materials and Methods}

Cell culture. $\mathrm{EpH} 4$ cells, a normal mouse mammary epithelial cell line, were grown in Dulbecco's modified Eagle's medium (DMEM) supplemented with $5 \%$ heat-inactivated fetal bovine serum (FBS), $1 \%$ penicillin/streptomycin, and $10 \mathrm{mM}$ Hepes (Mediatech, Manassas, VA, USA). Growth media were refreshed every 3-5 days and cells were trypsinized ( $0.25 \%$ trypsin, EDTA, Mediatech) and plated $1: 2$ every 7 days. Cells were plated with a 1:1 surface area onto Lab-Tek glass 8chamber slides (NUNC, Rochester, NY, USA) for experiments.

Primary mammary epithelial cells were isolated from the mammary glands of occludin wild-type $(+1+)$ FVB mice (Jackson Laboratories, Bar Harbor, ME, USA) and occludin knockout $(-/-)$ mice (kindly provided by Mikio Furuse, Kyoto University, Japan ${ }^{25}$ ) back-crossed onto FVB. The fourth and fifth mammary glands were dissected from pregnancy day-15 dams. Minced glands from the same mice were placed in collagenase solution consisting of DMEM/F12 media (Mediatech), $2 \mathrm{mg} / \mathrm{ml}$ collagenase A (Roche Applied Science, Indianapolis, IN, USA), and $50 \mu \mathrm{g} /$ $\mathrm{ml}$ gentamycin (Mediatech). Tissue, in collagenase solution, was incubated at $37^{\circ} \mathrm{C}$ with shaking at 200 r.p.m. for $2 \mathrm{~h}$. Cells were then spun at 1500 r.p.m. for $10 \mathrm{~min}$ and the pellet was pulse washed with phosphate buffered saline (PBS, with calcium and magnesium) 5 to 10 times at 1500 r.p.m. for $2 \mathrm{~s}$. The pellet was then resuspended in growth media containing DMEM/F12 media, $1 \times$ ITS $(\times 10$ stock, Sigma, St. Louis, MO, USA), $100 \mu \mathrm{g} / \mathrm{ml}$ epidermal growth factor (BD Biosciences, San Diego, CA, USA), 5\% FBS (Mediatech), $50 \mu \mathrm{g} / \mathrm{ml}$ gentamycin (Mediatech), 1\% penicillin/ streptomycin (Mediatech), and $2.5 \mu \mathrm{g} / \mathrm{ml}$ FUNGIZONE (Gibco, Grand Island, NY, USA), and plated on 8-well chamber slides coated with collagen (Sigma).

Immunofluorescence. Cell monolayers were fixed with $2 \%$ paraformaldehyde for $15 \mathrm{~min}$ at room temperature after treatment with the $\mathrm{D}$-form of a claudin mimic peptide (D-DFYNP, synthesized by the Peptide and Protein Chemistry Core, University of Colorado Denver, School of Medicine, Aurora, CO, USA ${ }^{14}$ ) or an occludin mimic peptide (LYHY, Peptide and Protein Chemistry Core ${ }^{13}$ ). Cells were then permeabilized with $0.5 \%$ Triton $X-100$ for 5 min before blocking with $2 \%$ bovine serum albumin for $1 \mathrm{~h}$. Cells were treated with mouse anti-claudin-4 (1:200, Zymed, Carlsbad, CA, USA), rabbit anti-claudin-4 (1:200, Zymed), rabbit anti-occludin (1:100, Invitrogen, Camarillo, CA, USA), rabbit anti-cleaved caspase-3 $(1: 100$, Cell Signaling Technologies, Danvers, MA, USA), rat cleaved caspase-8 $(1: 100$, Enzo Life Sciences, San Diego, CA, USA), mouse anti-FADD (1:100, BD Biosciences), mouse anti-Fas (1:100, Santa Cruz Biotechnology, Santa Cruz, CA, USA) and/or rat anti-ZO-1 (1:50, Santa Cruz Biotechnology) primary antibodies for $1 \mathrm{~h}$. After washing with PBS, cells were treated with donkey anti-mouse-CY5, donkey anti-mouse-FITC, donkey anti-rabbit-CY3, donkey anti-rat-FITC (1:150, Jackson ImmunoResearch Laboratories, West Grove, PA, USA) for $45 \mathrm{~min}$. Monolayers were then washed five times, $5 \mathrm{~min}$ each, with PBS and OPDA ( $20 \mathrm{mg} / \mathrm{ml}$ o-phenylenediamine dihydrochloride in $1 \mathrm{M}$ Tris, $\mathrm{pH}$ 8.5) was applied before addition of a coverslip. Fluorescence was imaged on an Olympus Spinning Disk confocal microscope, using SlideBook software (Intelligent Imaging Innovations, Inc., Denver, CO, USA).

Statistics. Data are presented as means \pm S.E.M. An unpaired Student's $t$-test was used for statistical comparison between control and treatment groups. A $P$-value of $<0.05$ was considered significant.

\section{Conflict of Interest}

The authors declare no conflict of interest.

Acknowledgements. We wish to thank Margaret C Neville, University of Colorado Denver, for her invaluable advice and encouragement throughout the study and during the preparation of this manuscript. We also thank Dziuleta Cepeniene, Peptide and Protein Chemistry Core, University of Colorado Denver, for synthesizing the mimic peptide used in this study and M Furuse, Kyoto, Japan for providing the occludin null-transgenic mouse. Financial support for this project was provided by Department of Defense Postdoctoral Fellowship Award W81XWH-09-10545 to HKB. We would also like to acknowledge that imaging was performed in the University of Colorado Advanced Light Microscopy Core, supported in part by NIH/ NCRR Colorado CTSI grant UL1 RR025780.

1. Liu S, Yang W, Shen L, Turner JR, Coyne CB, Wang T. Tight junction proteins claudin-1 and occludin control hepatitis $C$ virus entry and are downregulated during infection to prevent superinfection. J Virol 2009; 83: 2011-2014.

2. Evans MJ, von Hahn T, Tscherne DM, Syder AJ, Panis M, Wolk B et al. Claudin-1 is a hepatitis $C$ virus co-receptor required for a late step in entry. Nature 2007; 446 801-805.

3. Bruewer M, Utech M, Ivanov Al, Hopkins AM, Parkos CA, Nusrat A. Interferon-gamma induces internalization of epithelial tight junction proteins via a macropinocytosis-like process. FASEB J 2005; 19: 923-933.

4. Prasad S, Mingrino R, Kaukinen K, Hayes KL, Powell RM, MacDonald TT et al. Inflammatory processes have differential effects on claudins 2, 3 and 4 in colonic epithelial cells. Lab Invest 2005; 85: 1139-1162.

5. Martin TA, Jiang WG. Loss of tight junction barrier function and its role in cancer metastasis. Biochim Biophys Acta 2009; 1788: 872-891.

6. Benedicto I, Molina-Jimenez F, Barreiro O, Maldonado-Rodriguez A, Prieto J, MorenoOtero $R$ et al. Hepatitis $C$ virus envelope components alter localization of hepatocyte tight junction-associated proteins and promote occludin retention in the endoplasmic reticulum. Hepatology 2008; 48: 1044-1053.

7. Sonoda N, Furuse M, Sasaki H, Yonemura S, Katahira J, Horiguchi Y et al. Clostridium perfringens enterotoxin fragment removes specific claudins from tight junction strands: evidence for direct involvement of claudins in tight junction barrier. J Cell Biol 1999; 147: 195-204.

8. Lapointe TK, O'Connor PM, Jones NL, Menard D, Buret AG. Interleukin-1 receptor phosphorylation activates Rho kinase to disrupt human gastric tight junctional claudin-4 during Helicobacter pylori infection. Cell Microbiol 2010; 12: 692-703.

9. Kirschner N, Poetzl C, von den Driesch P, Wladykowski E, Moll I, Behne MJ et al. Alteration of tight junction proteins is an early event in psoriasis: putative involvement of proinflammatory cytokines. Am J Pathol 2009; 175: 1095-1106.

10. Bantel H, Schulze-Osthoff K. Apoptosis in hepatitis $\mathrm{C}$ virus infection. Cell Death Differ 2003; 10 (Suppl 1): S48-S58.

11. Moss SF, Calam J, Agarwal B, Wang S, Holt PR. Induction of gastric epithelial apoptosis by Helicobacter pylori. Gut 1996; 38: 498-501.

12. Schulzke JD, Ploeger S, Amasheh M, Fromm A, Zeissig S, Troeger $\mathrm{H}$ et al Epithelial tight junctions in intestinal inflammation. Ann NY Acad Sci 2009; 1165 294-300.

13. Beeman NE, Baumgartner HK, Webb PG, Schaack JB, Neville MC. Disruption of occludin function in polarized epithelial cells activates the extrinsic pathway of apoptosis leading to cell extrusion without loss of transepithelial resistance. BMC Cell Biol 2009; 10: 85

14. Baumgartner HK, Beeman N, Hodges RS, Neville MC. A d-peptide analog of the second extracellular loop of claudin-3 and -4 leads to mislocalized claudin and cellular apoptosis in mammary epithelial cells. Chem Biol Drug Des 2011; 77: 124-136.

15. Saitou M, Fujimoto K, Doi Y, Itoh M, Fujimoto T, Furuse $M$ et al. Occludin-deficient embryonic stem cells can differentiate into polarized epithelial cells bearing tight junctions. J Cell Biol 1998; 141: 397-408.

16. Tobioka H, Isomura H, Kokai Y, Tokunaga Y, Yamaguchi J, Sawada N. Occludin expression decreases with the progression of human endometrial carcinoma. Hum Pathol 2004; 35: 159-164.

17. Martin TA, Mansel RE, Jiang WG. Loss of occludin leads to the progression of human breast cancer. Int J Mol Med 2010; 26: 723-734.

18. Osanai M, Murata M, Nishikiori N, Chiba H, Kojima T, Sawada N. Occludin-mediated premature senescence is a fail-safe mechanism against tumorigenesis in breast carcinoma cells. Cancer Sci 2007; 98: 1027-1034.

19. Nusrat A, Parkos CA, Verkade P, Foley CS, Liang TW, Innis-Whitehouse W et al. Tight junctions are membrane microdomains. J Cell Sci 2000; 113 (Part 10): 1771-1781.

20. Lambert D, O'Neill CA, Padfield PJ. Methyl-beta-cyclodextrin increases permeability of Caco-2 cell monolayers by displacing specific claudins from cholesterol rich domains associated with tight junctions. Cell Physiol Biochem 2007; 20: 495-506. 
21. Algeciras-Schimnich A, Shen L, Barnhart BC, Murmann AE, Burkhardt JK, Peter ME. Molecular ordering of the initial signaling events of CD95. Mol Cell Biol 2002; 22: 207-220.

22. Eramo A, Sargiacomo M, Ricci-Vitiani L, Todaro M, Stassi G, Messina CG et al. CD95 death-inducing signaling complex formation and internalization occur in lipid rafts of type I and type II cells. Eur J Immunol 2004; 34: 1930-1940.

23. Zeissig S, Burgel N, Gunzel D, Richter J, Mankertz J, Wahnschaffe U et al. Changes in expression and distribution of claudin 2, 5 and 8 lead to discontinuous tight junctions and barrier dysfunction in active Crohn's disease. Gut 2007; 56: 61-72.

24. Jones NL, Shannon PT, Cutz E, Yeger H, Sherman PM. Increase in proliferation and apoptosis of gastric epithelial cells early in the natural history of Helicobacter pylor infection. Am J Pathol 1997; 151: 1695-1703.
25. Saitou M, Furuse M, Sasaki H, Schulzke JD, Fromm M, Takano $\mathrm{H}$ et al. Complex phenotype of mice lacking occludin, a component of tight junction strands. Mol Biol Cell 2000; 11: 4131-4142.

Cell Death and Disease is an open-access journal published by Nature Publishing Group. This work is licensed under the Creative Commons Attribution-Noncommercial-No Derivative Works 3.0 Unported License. To view a copy of this license, visit http://creativecommons.org/licenses/by-nc-nd/3.0/ 